Instituto Internacional de Investigación y Desarrollo Tecnológico Educativo INDTEC, C.A. DOI: https://doi.org/10.29394/Scientific.issn.2542-2987.2020.5.15.3.67-86

OAI-PMH: http://www.indteca.com/ojs/index.php/Revista Scientific/oai

Artículo Original / Original Article

\title{
Mirada Compleja y Transdisciplinaria del Objeto de Estudio de las Ciencias Organizacionales
}

\author{
Autora: Auresnelly Maribel Torres Triana \\ Universidad de los Andes, ULA \\ auresnelly@gmail.com \\ Mérida, Venezuela \\ https://orcid.org/0000-0002-7541-1408
}

\section{Resumen}

El objeto de estudio de las ciencias organizacionales del todo en la organización, visto de forma unitaria y no como partes aisladas del todo, por lo tanto, de acuerdo al criterio previo de la investigadora, las organizaciones no deben vulnerar la importancia de interrelación y complementariedad que se produce como resultado de la realidad de donde es envuelta y es llevado a cabo todos los procesos y actividades del ser humano, con todo lo que lo rodea. Por consiguiente, se presenta como propósito general analizar sobre el objeto de estudio de las ciencias organizacionales desde la mirada del paradigma complejo y transdiciplinario. En este mismo orden de ideas, se considera el estudio desde el diseño de investigación documental y paradigma interpretativo, puesto que en base a la fundamentación teórica desarrollada durante la investigación se consideran aspectos teóricos y postulados teóricos que permitieron realizar un proceso de análisis e interpretación a través de una matriz ilustrativa que indicará el resultado de la fundamentación teórica abordada en la investigación. Por lo que como principal reflexión investigativa se resalta la significancia de que las organizaciones son entidades humanas y sociales, sistemas abiertos, flexibles que integra e interrelacionan, el paradigma, complejo y transdiciplinario.

Palabras clave: organización; sociedad; empresa.

Cómo citar este artículo:

Torres, A. (2020). Mirada Compleja y Transdisciplinaria del Objeto de Estudio de las Ciencias Organizacionales. Revista Scientific, 5(15), 67-86, e-ISSN: 2542-2987. Recuperado de: https://doi.org/10.29394/Scientific.issn.2542-2987.2020.5.15.3.67-86

Fecha de Recepción:

24-06-2019
Fecha de Aceptación:

30-09-2019
Fecha de Publicación: 05-02-2020 


\title{
Complex and Transdisciplinary Look at the Object of Study of Organizational Sciences
}

\begin{abstract}
The object of study of the organizational sciences of the whole in the organization, seen as a unit and not as isolated parts of the whole, therefore, according to the previous criteria of the researcher, organizations should not violate the importance of interrelation and complementarity that occurs as a result of the reality of where it is wrapped and is carried out all the processes and activities of the human being, with everything that surrounds it. Therefore, it is presented as a general purpose to analyze the object of study of the organizational sciences from the perspective of the complex and transdisciplinary paradigm. In this same order of ideas, the study is considered from the design of documentary research and interpretive paradigm, since based on the theoretical foundation developed during the investigation, theoretical aspects and theoretical postulates that allowed to perform a process of analysis and interpretation to through an illustrative matrix that will indicate the result of the theoretical foundation addressed in the research. Therefore, the main investigative reflection highlights the significance that organizations are human and social entities, open, flexible systems that integrate and interrelate, the paradigm, complex and trans-disciplinary.
\end{abstract}

Keywords: organization; society; enterprise.

How to cite this article:

Torres, A. (2020). Complex and Transdisciplinary Look at the Object of Study of Organizational Sciences. Revista Scientific, 5(15), 67-86, e-ISSN: 2542-2987. Recovered from: https://doi.org/10.29394/Scientific.issn.2542-2987.2020.5.15.3.67-86

Date Received:

24-06-2019
Date Acceptance:

30-09-2019
Date Publication: 05-02-2020 


\section{Introducción}

El hombre vive en una sociedad de organizaciones en donde todo lo que produce y necesita se encuentra dentro de ellas, cubriendo en su totalidad todas las necesidades de consumo del individuo tanto material como inmaterial. En este sentido, según las posturas de pensamiento de Aristóteles, Platón y Husserl, dicen en base a Pantoja y Zuñiga (1995), que el todo es un:

Conjunto de partes, independiente de la ordenación de estas; lo que puede ser descompuesto o dividido en sus partes. Aristóteles formuló como todo a aquella colectividad en el cual no hace falta ninguna de sus partes conformantes o aquello que tiene sus partes constitutivas como unidad. Para Platón el todo es una entidad estructurada en donde cada componente ocupa un lugar preciso, y del simple hecho de modificar el lugar, da como resultado la alteración del sistema en forma integral. Para Husserl, todo es el conjunto de contenidos cubiertos en una fundamentación unitaria, es decir, qué contenido está por base directa o indirectamente con algún otro contenido (pág. 480).

De acuerdo con lo anteriormente planteado, se analiza e interpreta que la organización es vista como un conjunto de partes en el que el hombre forma parte de sus partes como un todo, de manera integral, por lo que, los seres humanos se organizan para lograr sus propios objetivos y los objetivos de la organización, lo que constituye una unidad del todo de las partes, para lo que Edmund Husserl (1859-1938) denomina fundamentación Unitaria.

En este orden de ideas, Rojas (2014a), dice que para Husserl: "de acuerdo con sus planteamientos, existe un mundo científico-objetivo que es una matematización e idealización del mundo vital, del mundo real, ese mundo pre-dado que percibimos y experimentamos. La ciencia se construye sobre ese mundo (...)" (pág. 33). Por otra parte, desde el contexto organizacional, Chiavenato (2002a), expone que: “(...) las organizaciones invaden nuestra sociedad (...) y una organización es una sociedad en miniatura" (pág. 10).

No obstante, a partir de la ciencia administrativa es importante señalar 
los grandes aportes y avance de la ciencia en este campo, porque corresponden a la edificación del paradigma clásico - moderno de la organización, en este sentido, Rodríguez (2015a), expone: que no podemos olvidar la teoría administrativa desde sus inicios de donde realmente surge el fenómeno organizacional en sus diferentes enfoques o paradigmas, en función de las generalidades de la teoría de Frederick Taylor (Administración Científica), Henri Fayol (Principios y funciones de la administración), Elton Mayo (teoría de las relaciones humanas/Hawthorne Studities) (pág. 19-28).

Por lo tanto, se analiza e interpreta previamente acerca de estos aportes científicos que los diferentes enfoques teóricos han contribuido a desarrollar la razón de ser de las organizaciones, por lo que es necesario considerar estos enfoques teóricos, dando pasos acelerados a la complementariedad e interacción del surgimiento de nuevos paradigmas del conocimiento, como lo son paradigma complejo y transdiciplinario en el objeto de estudio de la ciencia organizacional, puesto que corresponden en este estudio a un punto de inflexión de la historia administrativa de la humanidad y por ende de la sociedad, siendo necesario hoy en día analizar. Del mismo modo, el estudio de la complejidad en términos de Morin (2005a), concibe que:

No hace falta creer que la cuestión de la complejidad se plantea solamente hoy en día, a partir de nuevos desarrollos científicos. Hace falta ver la complejidad allí donde ella parece estar, por lo general, ausente, como, por ejemplo, en la vida cotidiana (pág. 54).

Asimismo, desde la transdisciplinariedad se ha devenido, desde mediados del Siglo XX, convirtiéndose en objeto de estudio esencial para la ciencia contemporánea, así como también para la reflexión filosófica, ética y social de la organización. Originando, que hoy día se considere el acaecimiento de que las diferentes disciplinas científicas están llamadas a buscar y a través de una racionalidad, los diversos elementos y aspectos de 
las ciencias humanas que trascienden más allá de lo disciplinar; no obstante, las disciplinas deben avocarse a encontrar una racionalidad diversa, que esté permeada por el propio paradigma de la complejidad, lo que se conlleva en términos de la ciencia organizacional involucrar la transdisciplinariedad, por lo que la visión del carácter transdiciplinario desde la complejidad, debe ser percibida en los estilos gerenciales que conviven en el seno de las organizaciones, como enfoque científico de la organización.

De esta manera en términos de la complejidad y la transdisciplinariedad, se encuentran presentes en general durante el surgimiento del pensamiento organizacional, puesto que su comprensión filosófica, desde su comienzo, es compleja, es decir, parte del entendimiento global del fenómeno y de sus propias percepciones, para después ser interpretados de acuerdo a su realidad (fenómeno)estudiado.

\section{EI Problema}

La ciencia administrativa indica que el objeto de estudio de la ciencia organizacional se cimienta desde el paradigma clásico-moderno, Rojas (2014b), dice: "a partir de las publicaciones de Khun, el termino paradigma ha provocado numerosas reflexiones en torno a su significado y alcances. Aparentemente, el paradigma representa una manera de observar al mundo, de comprender y explicar la realidad" (pág. 19).

En este sentido, el objeto de estudio de la organización, se enfoca desde la visión clásica en función de métodos, reglas, jerarquías, control, división del trabajo, impersonalidad, comunicaciones formales, reglamentaciones, rigidez, esto quiere decir, en función de objetivos organizacionales administrativos, económicos y financieros, por lo que es visto desde términos de rentabilidad, donde el ser humano es contemplado y tratado en función de su fuerza de trabajo (llámese cognitivas o físicas) mas no como ser que tiene comportamientos y conductas distintas. 
Por su parte, desde la visión moderna, Elton Mayo (1880-1949) fue el pionero en denominar a los trabajadores como "Recurso Humano" en función del estudio aplicado a las relaciones de trabajo junto con el comportamiento grupal de los trabajadores.

Desde este contexto, el criterio de la investigación se orienta a analizar ¿Cómo y Por qué? el objeto de estudio de las ciencias organizacionales desde la mirada del paradigma complejo y transdiciplinario, por lo que se considera la posibilidad de ser un enfoque integrador, es decir, se determina una interpretación integradora del fenómeno organizacional, puesto que el proceso de globalización como lo denomina Edgar Morin "planetarización", la era digital, las Tecnologías de Información y la Comunicación (TIC) las ciencias humanas, la explotación de los recursos, los daños ambientales, el desarrollo y crecimiento de la población, en si todo los problemas que rodea, atañe a la humanidad, son cada vez más caóticas, complejas de estudiar y comprender de forma individual y aislada, debido que las cosas en sí mismas integran una realidad por las interacciones propias de cada campo de acción así como de transformación en el que se encuentran y entrecruzan.

Por lo tanto, en estos términos las organizaciones se muestran hacia las praxis y el conocimiento de integrar nuevos procesos y estilos gerenciales que complemente e integren y junten lo tradicional y clásico con lo flexible, humano, complejo, transdiciplinario, para ir hacia la búsqueda de la construcción de nuevas formas de organización, de acuerdo con el contexto que correspondan analizarlas.

No obstante, la situación problemática se centra en el objeto de estudio de las ciencias organizacionales de hoy; por lo que se contribuye al acerco del conocimiento organizacional vista desde el paradigma complejo y transdiciplinario. Se analiza que, a través de los avances de la ciencia, las exigencias, desafíos, e incertidumbre y paradigmas de la organización actual, se conducen a la solución de problemas humanos y sociales de índole integral- 
complementario en donde el hombre se desenvuelve, actúa y se organiza para la satisfacción de necesidades humanas, colectivas, sociales como un todo, y no visto de forma aislada el hombre (sujeto) y la organización (objeto).

Por otra parte, el problema implica en términos filosóficos y epistemológicos en no ver el objeto de la organización como la suma de las partes que conforman el todo sino como el todo de las partes de la ciencia organizacional, por lo que representará integrar a la ciencia organizacional todos los aspectos tanto internos como externos que rodean al hombre y a la organización así como interpretar el fenómeno complejo en la cual se encuentra envuelta, por lo que la investigadora se sustentará en los planteamientos de autores relacionados con la temática esbozada. En este sentido, surgen las siguientes inquietudes: ¿Qué relación existe en el objeto de estudio de la ciencia organizacional vista desde las partes son el todo y el todo son las partes? ¿Hacia dónde debe ir hoy dirigido el objeto de estudio de las ciencias organizacionales? Desde este contexto, el propósito de la investigación corresponde en: analizar sobre el objeto de estudio de las ciencias organizacionales desde la mirada del paradigma complejo y transdiciplinario.

\section{Referentes Teóricos}

\subsection{Organización}

En términos del paradigma clásico de la organización, se analiza e interpreta como objeto de estudio "las organizaciones" fundamentada específicamente por la necesidad de aumentar la eficiencia de las organizaciones, con el propósito de obtener el mejor rendimiento posible de sus recursos, por lo que a través del desarrollo del objeto de estudio de la organización se presenta:

La Teoría de Frederick Taylor (1856-1915), quién desarrolló inicialmente la llamada escuela científica del pensamiento administrativo 
"Administración Científica" vista en términos de aumentar la eficiencia de la organización a través de la racionalización del trabajo en base a los niveles de productividad del trabajador.

Por su parte, en cuanto a la teoría clásica de Henry Fayol (1841-1925), se ocupó también del aumento de la eficiencia de la organización a diferencia de Taylor mediante la teoría de la administración científica conocida como fayolismo, asimismo desarrolló la clasificación y aplicación de los principios generales y básicos de la administración: planificación, organización, dirección, coordinación y control; por lo que sus ideas constituyen la base del conocido enfoque clásico de la administración cuyos argumentos dominaron el objeto de estudio de la ciencia organizacional durante las cuatro primeras décadas del Siglo XX.

De igual forma, en términos del paradigma moderno, la teoría de las relaciones humanas de Elton Mayo (1880-1949), surge para la cuarta década del Siglo XX, a través del desarrollo de las ciencias sociales, por lo que hoy en día se considera los grandes aportes científicos que hace esta teoría en función del recurso humano en una organización, asimismo fue quien condujo el famoso Experimento de Hawthorne, para lo que se antepone a la teoría de los clásicos, por lo que el objeto de estudio de la ciencia organizacional desde esta teoría se considera al trabajador como "factor humano" de gran importancia dentro de los procesos organizacionales. En este sentido, se enfoca desde el principio de dirección, donde se reconoce el factor humano por lo que en base a Koontz y Weihrich (2007), dicen que se:

Requiere que se cree y sostenga un ambiente en el cual los individuos colaboren en grupo para alcanzar los objetivos comunes (...) se subraya la importancia de conocer y aprovechar los factores humanos y de motivación (...) el trabajo del líder o jefe no es manipular a las personas sino reconocer qué las motiva (pág. 282).

Desde esta concepción teórica, en función de la teoría de las relaciones 
humanas, Cejas y Jácome (2006), citados en Espinoza (2009):

Ya han señalado que en esta época de cambio la organización ha ido superando la estructura jerárquica y rígida. La organización de hoy va evolucionando hacia formas organizacionales basadas en la producción ligera, la flexibilidad y la comunicación. La nueva organización hace énfasis en los equipos interfuncionales (pág. 61).

En este orden de ideas, de acuerdo a lo planteado en el paradigma clásico y moderno, el objeto de estudio de las ciencias organizacionales, se analiza: que para llevar a cabo el estudio de las organizaciones actualmente en base a su objeto científico, se plantea previamente por parte de la investigadora, que desde este enfoque, es necesario considerar y desarrollar hoy en día la búsqueda de una nueva visión organizacional por lo que Gil (2013a), dice que: "el paradigma de la complejidad que hoy nos arropa, nos permite afirmar que las partes son el todo y el todo es las partes, aunque el todo no es la suma de las partes sino mucho más" (pág. 21).

Desde este contexto, a partir del hilo epistemológico de hoy, la interpretación y análisis del fenómeno objeto de estudio de la organización corresponden a un sistema de carácter inductivo, abierto y flexible porque para este estudio se aborda sobre el criterio de las ciencias organizacionales desde la mirada del paradigma complejo y transdiciplinario. En este sentido, lo que se concibe en esta investigación hace referencia a que las organizaciones interactúan con un entorno externo de diversas variables: ambientales, demográficas, políticas, sociales, económicas, entre otras, de esta manera, se involucran factores internos que son propicios dentro del contexto de la organización, apuntándose así, a un fenómeno organizacional que se ocasionan dentro y fuera de la organización, por lo que se infiere que entre las diversas relaciones con el entorno (interno-externo) dependerá el objeto de estudio de la organización, así como de las exigencias, desafíos e incertidumbre, necesidades, que se esperan satisfacer a nivel humano y 
social.

Por lo tanto, estas relaciones muestran cómo se requiere de un sistema abierto y flexible para recibir del entorno (ambiente) las diversas condiciones naturales externas que se transforman en el origen del producto de cambio, los cuales regresan al entorno, pudiendo algunos de esos elementos volverlos al sistema. Para Chiavenato (2002b): "las organizaciones son sistemas abiertos que interactúan dinámicamente con sus ambientes. Un sistema abierto es aquel que mantiene intensa interacción (entradas y salidas) con su medio, afectándolo y siendo afectado por éste" (pág. 76). De igual forma, es necesario analizar acerca de la teoría del sistema abierto en base a Katz y Kahn (2008a), por lo que conciben:

La teoría del sistema abierto, con su supuesto de la entropía, hace hincapié en la íntima relación que existe entre una estructura y el ambiente que le da apoyo, pues de no haber insumos continuos, pronto la estructura se va a pique. De este modo, una base crítica para identificar sistemas sociales la constituyen sus relaciones con las fuentes energéticas que los mantienen. (...) (pág. 17).

Seguidamente, las organizaciones, refiriendo a Pastor y García (2007): deben ser percibidas como sistemas adaptativos complejos, pues son sistemas autónomos, la autonomía de los elementos del sistema se muestran en la adaptabilidad, la transformación, la mutación, el desarrollo de los procesos correctivos de que se proceden de la sistematización, precisamente en las características o propiedades que deben tener los recursos o entradas que son percibidas del entorno externo de la organización, conjuntamente son autoorganizados y autosostenidos, productos propio del mismo sistema, con extensos periodos de estabilidad, dispersos en su estructura y funcionamiento que, incomprensiblemente, permiten que estas organizaciones se estabilicen, puesto que se trata de sistemas coherentes, aunque no homogéneos; sistemas caóticos y complejos, pero ordenados en ese mismo desbarajuste 
que posibilita que los elementos se reestructuren de acuerdo con las influencias o necesidades de los ambientes (entornos) internos y/o externos de las organizaciones (pág. 214).

\subsection{Complejidad}

Iniciando en el campo de estudio del objeto de las organizaciones, la ciencia clásica sintió sus bases científicas en términos de la Administración Científica desde el paradigma Taylorista, asimismo desde el pensamiento de Henri Fayol, dando una conceptualización científica al término, así como las funciones y procesos de la administración como parte de una organización, seguidamente desde la crítica de pensamiento moderno de la administración se antepone el pensamiento de Mayo con una significada importancia del trabajador en la organización, por supuesto sin dejar de lado que cada uno de ellos condujeron y aportaron "ciencia" desde su contexto histórico, económico y social de cada época, hasta lo que ahora hoy en día ha sido el conocimiento científico de la organización.

En este sentido, la vida social se ha ido complejizando y la sociedad se ha ido transformando y cambiando de acuerdo a sus épocas y acontecimientos, donde el ser humano cada día demanda satisfacer a gran escala sus necesidades e incertidumbres, en la cual es importante enfrentar retos así como desafíos que presenta la humanidad, por lo que se debe considerar la interconexión de aspectos sociales, políticos, económicos, ecológicos con el ser humano y su entorno interno-externo, sumergido en una realidad, por lo que Gil (2013b), denomina:

(...) realidad fenomenológica que hace de las partes constituyentes fundamentales un todo, pero ese todo es al mismo tiempo las partes. Ya los fenómenos no son vistos como la sumatoria de unos compartimientos desconectados, independientes, estancos, sino como procesos integrales e integrados en cuya comprensión intervienen factores, elementos, vasos comunicantes con la realidad física y con el 
universo (pág. 20).

Por consiguiente, desde esta visión fenomenológica las organizaciones constituyen un todo, representado en un paradigma complementario, transdiciplinario y complejo sobre la realidad, la vida y el hombre, por lo que se requiere un acercamiento también totalizador y situado en nuestra propia realidad, por ello, Gil (2013c), dice que: "la complejidad resulta una respuesta significativa a fenómenos que coexisten en nuestra realidad, pero que no pueden ser explicados por las vías tradicionales (...)" (pág. 19).

Por otro parte, Rodríguez y Leónidas (2011), en su investigación sobre las Teorías de la complejidad y ciencias sociales, Nuevas Estrategias Epistemológicas y Metodológicas, concluyen que:

(...) el pensamiento complejo se afirma como una concepción epistémica alternativa al tradicional modo de entender la ciencia y el conocimiento. Su estrategia metodológica se distancia del concepto estándar de método científico, para reclamar la necesidad y pertinencia de un método que incluya la reflexión crítica y auto-crítica; es decir, postula la inclusión del sujeto cognoscente en su conocimiento (...) (pág. 17).

En base a lo planteado por los autores, quiere decir que el pensamiento complejo representa un salto científico importante que implica incluir en la comprensión del conocimiento científico, sin dejar a un lado el objeto central de la ciencia clásica y moderna sino al contrario el pensamiento complejo debe ser articulada e integrada con otras formas de conocimiento científico.

\subsection{Transdisciplinariedad}

De acuerdo a lo expuesto desde la complejidad, lo que se debe cambiar y transformar es la prospectiva de la organización de hoy, desde la visión del todo en el que convergen todas las entidades sociales y al hombre, puesto que se complementan e integran hacia la transdisciplinariedad organizacional que 
quiere decir mirar más allá de las partes constituyentes de un todo.

Desde este paradigma transdiciplinario, en base a Ander-Egg (1999), citado en Rodríguez (2015b), expresa que: “(...) la transdisciplinariedad se trata de un nivel máximo de integración, en donde se borran las fronteras entre disciplinas" (pág. 74). Esto quiere decir, es que la organización no aborde ni enfrente los problemas organizacionales de forma individual o como una parte, sino que tiene y debe tomar en cuenta el todo, (su entorno, ambiente, variables sociales, familiares, éticos, religiosos, culturales, demográficos, otros), puesto que, no debe estudiar un solo elemento o un solo campo de la ciencia, una única disciplina o única postura de pensamiento, sino que debe involucrar otros elementos y variables externas, los cuales se necesitan complementar, e ir más allá de lo tradicional.

Del mismo modo, que corresponda la integración de todos los campos de la ciencia y del saber, es decir, hacia la transdisciplinariedad de: las ciencias administrativas, ciencias sociales, ciencias jurídicas, ciencias de la salud, sociología, psicología, filosofía, educación, ecología así como el fundamento ético y moral del individuo (conductas, comportamientos, estilos de pensamiento), esto quiere decir con todas las disciplinas de la ciencia y conocimientos se complementan e integran como un todo para el desenvolvimiento, y desarrollo de la organización.

También desde la transdisciplinariedad de la organización se considera la producción de nuevo conocimiento en la ciencia organizacional, la cual proviene de la posición espiritual y cognitiva del ser humano así como de los recurrentes escenarios de transformación de la sociedad, por lo que desde el terreno de la transdisciplinariedad se visualiza, como una nueva forma de organización que trasciende más allá de la interdisciplinariedad, se trata de extender y complementar epistemologías que convergen y emergen en el campo de la investigación científica, lo cual conlleva a una organización transcompleja que logre integrar los objetivos organizacionales con los 
individuales de acuerdo a las necesidades tanto humanas como colectivas y sociales, permitiendo así unificar relaciones dialógicas, información, comunicación, tecnologías, diseños, investigación, innovación y creación de nuevos conocimientos para proporcionar respuestas asertivas hacia el logro de necesidades del ser humano y de la organización.

En este sentido, se interpreta y analiza que el objeto de estudio de las ciencias organizacionales de hoy, es el objeto y sujeto donde se llevan a cabo todas las actividades del hombre inclusive desde el comportamiento, conducta, conocimientos, como ser y ente de la organización, el incentivo del trabajo en equipo, el espíritu colaborativo de innovación, el tipo de liderazgo, la motivación, el salario emocional, el valor al trabajo, la creación de conocimientos, la competitividad empresarial, la creación e innovación de organizaciones digitales que permitan ampliar e ir más allá la razón de ser de la organización vista en términos flexibles, humanos, materiales, financieros, en fin todo lo que corresponda al entorno interno-externo de la organización que involucra la sociedad, los cuales conducen a la visión de la organización como el todo que se interrelacionan, integran, todos los procesos y operaciones que llevan a cabo la actividad humana y la organización.

\section{Marco Metodológico}

El contexto metodológico de la presente investigación se encuentra enmarcado desde el diseño de investigación documental y del paradigma interpretativo, Piñero y Rivera (2013a), argumentan que este paradigma: "surge como un intento de superar la rigidez y la unilateralidad del positivismo. También llamado paradigma cualitativo, fenomenológico, naturalista, humanista o construccionista. Como puede observase las diferentes denominaciones que recibe" (pág. 31).

Asimismo, para Yuni y Urbano (2005), citados en Piñero y Rivera (2013b), expresan que: 
Obedece a la concepción filosófico de la que se nutre y que le provee los fundamentos acerca de lo "real" y las posibilidades de conocerlo, de igual forma corresponde a los supuestos metodológicos en los que basa su pretensión de elaborar una teoría de los social y finalmente el papel que los actores sociales ocupan en la producción y reproducción de lo social (pág. 31).

De acuerdo a lo planteado por los autores, la naturaleza interpretativa de la realidad corresponde a la profundidad de análisis que puede llegar a obtener una comprensión del comportamiento humano dentro del campo filosófico, enfoque epistemológico y su significado en la investigación científica. Asimismo, con respecto al diseño de investigación documental, el Manual de trabajos de grado de especialización y maestría y tesis doctorales de la Universidad Pedagógica Experimental Libertados (UPEL, 2011):

Se entiende por investigación documental, el estudio de problemas con el propósito de ampliar y profundizar el conocimiento de su naturaleza, con apoyo, principalmente, en trabajos previos, información y datos divulgados por medios impresos, audiovisuales o electrónicos. La originalidad del estudio se refleja en el enfoque, criterios, conceptualizaciones, reflexiones, conclusiones, recomendaciones y, en general, en el pensamiento del autor (pág. 20).

Por lo tanto, la investigación se sustentó en fuentes secundarias, artículos, trabajos de investigación, textos, documentos electrónicos de carácter científico, puesto que se argumenta y se analiza sistemáticamente en base a conceptualizaciones provenientes de los tipos de documentos previamente consultados, para así indagar sobre el objeto de estudio, aportando nuevos conocimientos a la investigación a partir de dichas fuentes.

En este sentido, en la presente investigación se establece en el hecho de que el comportamiento humano es distinto, sus apreciaciones, sus percepciones en base a la realidad en el que se desenvuelve, produciéndose así un fenómeno, así como la naturaleza de la organización; por tal razón el 
paradigma cualitativo abarca la comprensión, la interpretación del estilo de pensamiento humano de acuerdo al contexto que le rodea, en función de sus conductas, limitaciones, opiniones, emociones, actitudes e interpretaciones de la realidad y del mundo. En este mismo orden de ideas, de acuerdo con lo que se observa y se experimenta en la sociedad y al fenómeno de la complejidad y transdisciplinariedad, se debe involucra cubrir los grandes desafíos, exigencias e incertidumbre de la humanidad, por lo que desde este escenario.

\section{Análisis e interpretación de los resultados}

Se concibe de forma ilustrativa y analítica el objeto de estudio de las ciencias organizacionales desde la mirada del paradigma complejo y transdiciplinario, los cuales corresponden a la interpretación de los hallazgos obtenidos por medio de la revisión documental realizada durante el estudio, en términos de que las organizaciones corresponden a un sistema abierto, flexible, complejo y transdiciplinario. Es importante, destacar que son cambiantes en función del comportamiento humano y social, vistas de forma integral y complementaria, como se puede apreciar en la figura 1.

Figura 1. Matriz ilustrativa del objeto de estudio de las ciencias organizacionales desde la mirada del paradigma complejo y transdiciplinario.

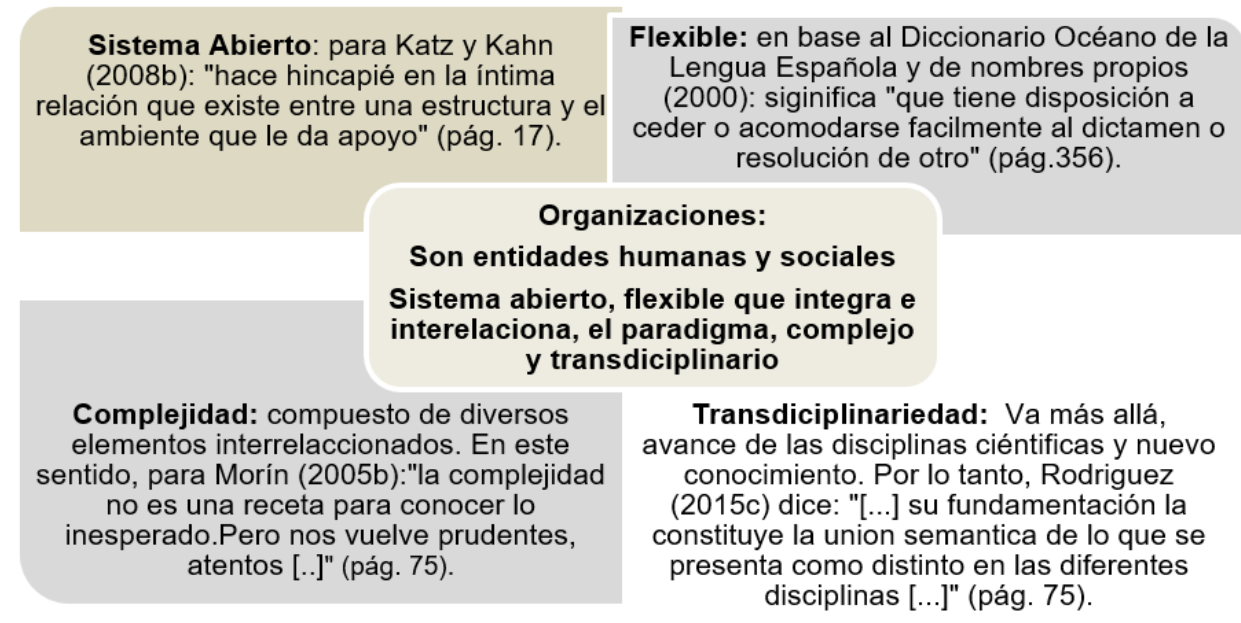

Fuente: La Autora (2019). 


\section{Reflexiones Finales}

Desde la mirada compleja y transdiciplinaria del objeto de estudio de las ciencias organizacionales, la epistemología de la organización cambia, es decir se transforma integrando e interrelacionando todos los elementos, factores y recursos necesarios que surgen de las necesidades del ser humano en el seno de la organización de acuerdo a los retos y desafíos que esta era planetaria demanda, puesto que es visto como un todo y no de forma aislada e independiente como la ciencia administrativa ha venido desarrollando desde sus inicios.

Puesto que hoy en día ya no resulta suficiente comprenderlo de tal forma, dado que es necesario ir más allá de la visión rígida y tradicional, porque permite la conformación de un sistema global que transforme las fronteras del conocimiento científico tradicional y disciplinario puesto que desde este contexto Rodríguez (2015d), dice que: “(...) existe la posibilidad de lograr modelos, principios y leyes que funcionan en distintos sistemas, mesosistemas y microsistemas, con la posibilidad de poder compartir teorías, conceptos y metodologías (...)" (pág. 74).

En este sentido, se pretende enfrentar las exigencias, desafíos, incertidumbres y necesidades del hombre y de la organización, porque tiene suficiente relevancia en la solución de los grandes problemas organizacionales de la humanidad, puesto que la ciencia avanza, avanza los modos de producción, avanzan las tecnologías, avanza la complejidad del comportamiento humano, y la ciencia organizacional, por lo tanto, se reflexiona en el sentido que le da Morin (2005c): este fenómeno, como "la relación antropo-social es compleja, porque el todo está en la parte, que está en el todo" (pág. 68); es decir, hacia la creación del conocimiento sobre el ser humano de forma integral en sus diversas formas con la sociedad, en este caso con la organización. 


\section{Referencias}

Chiavenato, I. (2002a,b). Administración en los nuevos tiempos. Bogotá, Colombia: McGraw-Hill.

Diccionario Océano de la Lengua Española (2000). Flexible. ISBN: 84-4942111-X. Barcelona, España: Editorial OCÉANO.

Espinoza, S. (2009). El fayolismo y la organización contemporánea. Visión Gerencial, (1), 53-62, e-ISSN: 1317-8822. Recuperado de:

https://www.redalyc.org/articulo.oa?id=465545880010

Gil, R. (2013a,b,c). Tiempos complejos ¿fin del método científico? Segunda Edición, ISBN: 978-980-11-1593-9. Mérida, Venezuela: Impresión Universidad de los Andes, Talleres gráficos universitarios.

Koontz, H., \& Weihrich, H. (2007). Elementos de administración. Un enfoque internacional. Séptima edición. México: McGraw-Hill.

Katz, D., \& Kahn, R. (2008a,b). Psicología social de las organizaciones. México: Editorial Trillas.

Morin, E. (2005a,b,c). Introducción al pensamiento complejo. México, D.F.: Multiversidad Mundo Real Edgar Morin. Recuperado de: http://cursoenlineasincostoedgarmorin.org/images/descargables/Morin Introduccion al pensamiento complejo.pdf

Pastor, J., \& García, A. (2007). Complejidad y psicología social de las organizaciones. Psicothema, 19(2), 212-217, e-ISSN: 0214-9915. Recuperado de: http://www.psicothema.com/psicothema. $a s p ? i d=3350$

Pantoja, L., \& Zuñiga, G. (Comp.) (1995). Diccionario Filosófico. Bogotá, Colombia: Nika Editorial, S.A.

Piñero, M., \& Rivera, M. (2013a,b). Investigación cualitativa: Orientaciones procedimentales. Colección General, ISBN: 978-980-7464-01-7. Barquisimeto, Venezuela: Universidad Pedagógica Experimental Libertador - UPEL.

Rojas, B. (2014a,b). Investigación Cualitativa: Fundamentos y praxis. 
ISBN: 980-273-471-3. Caracas, Venezuela: Fondo Editorial de la Universidad Pedagógica Experimental Libertador - FEDUPEL.

Rodríguez, M. (2015a,b,c,d). La gerencia interdisciplinaria lo natural y lo

humano. Valencia, Venezuela: Instituto de Previsión Social del Personal Docente y de Investigación de la Universidad de Carabobo IPAPEDI.

Rodríguez, L., \& Leónidas, J. (2011). Teorías de la complejidad y ciencias sociales. Nuevas Estrategias Epistemológicas y Metodológicas. Nómadas. Revista Crítica de Ciencias Sociales y Jurídicas, 30(2), 1-20, e-ISSN: 1578-6730. Recuperado de:

http://www.redalyc.org/articulo.oa?id=18120143010

UPEL (2011). Manual de Trabajos de Grado de Especialización y Maestría y Tesis Doctorales. 4ta Edición, Reimpresión, ISBN: 980-273-441-1. Caracas, Venezuela: Fondo Editorial de la Universidad Pedagógica Experimental Libertador, FEDUPEL. 


\section{Auresnelly Maribel Torres Triana \\ e-mail: auresnelly@gmail.com}

Nacida en Mérida, Venezuela, el 25 de agosto del año

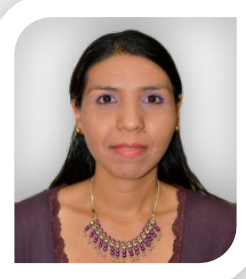
1981. Economista por la Universidad de Los Andes (ULA); Postgrado en Ciencias Contables de la Universidad de Los Andes (ULA); Componente Docente Básico en Educación Superior en la Universidad de Oriente (UDO); Funcionario Público de Carrera del Instituto Nacional de Desarrollo de la Pequeña y Mediana Industria (INAPYMI); Profesora Contratada de pregrado y postgrado de la Universidad Nacional Experimental de los Llanos Occidentales Ezequiel Zamora (UNELLEZ); Profesora invitada a nivel de pregrado, postgrado de en la Universidad Fermín Toro (UFT); y la Universidad Nacional Experimental de la Seguridad (UNES); Asesor y jurado de trabajos de investigación de la Universidad Fermín Toro (UFT); Doctorando en Ciencias Organizacionales de la Universidad de Los Andes (ULA), Mérida, Venezuela.

El contenido de este manuscrito se difunde bajo una Licencia de Creative Commons ReconocimientoNoComercial-Compartirlgual 4.0 Internacional 